\title{
A nonlinearly ill-posed problem of reconstructing the temperature from interior data *
}

\author{
Pham Hoang Quan ${ }^{\dagger}$ - Dang Duc Trong ${ }^{\dagger}$ \\ Alain Pham Ngoc Dinh
}

\begin{abstract}
We consider the problem of reconstructing, from the interior data $u(x, 1)$, a function $u$ satisfying a nonlinear elliptic equation

$$
\Delta u=f(x, y, u(x, y)), \quad x \in \mathbb{R}, y>0 .
$$

The problem is ill-posed. Using the method of Green function, the method of Fourier transforms and the method of truncated high frequencies, we shall regularize the problem. Error estimate is given.
\end{abstract}

Keywords Fourier transform, Contraction, ill-posedness.

AMS Classification 2000: 47J06, 35J60, 42A38, 47H10.

\section{Introduction}

In this paper, we consider the problem of reconstructing the temperature of a body from interior measurements. In fact, in many engineering contexts (see, e.g., [BBC]), we cannot attach a temperature sensor at the surface of a body (e.g., the skin of a missile). Hence, to get the temperature distribution on the surface, we have to use the temperature measured inside the body.

Precisely, we consider a two-dimensional body represented by the half-plane $\mathbb{R} \times \mathbb{R}^{+}$. Letting $u(x, y)$ be the temperature of the body at $(x, y) \in \mathbb{R} \times \mathbb{R}^{+}$and letting $f \equiv f(x, y, u)$ be a (nonlinear) heat source, we have the following nonlinearly nonhomogeneous equation

$$
\Delta u=f, \quad x \in \mathbb{R}, y>0
$$

where $\Delta=\frac{\partial^{2}}{\partial x^{2}}+\frac{\partial^{2}}{\partial y^{2}}$. We assume that the temperature on the line $y=1$ is known, i. e.,

$$
u(x, 1)=\varphi(x),
$$

* Supported by the Council for Natural Sciences of Vietnam

${ }^{\dagger}$ HoChiMinh City National University, Department of Mathematics and Informatics, 227 Nguyen Van Cu, Q. 5, HoChiMinh City, VietNam. Email: tquan@pmail.vnn.vn, ddtrong@mathdep.hcmuns.edu.vn

${ }^{\ddagger}$ Mathematics Department, Mapmo UMR 6628, BP 67-59, 45067, Orleans Cedex2, France. Email: alain.pham@math.cnrs.fr 
and that

$$
u(x, y) \rightarrow 0 \text { as }|x|, y \rightarrow \infty
$$

The problem can be referred as a sideways elliptic problem and the interior measurement $\varphi(x)$ is also called (in geology) the borehole measurement. The problem can be splitted into two problems:

Problem 1: finding the function $u$ satisfying

$$
\Delta u=f, \quad x \in \mathbb{R}, y>1
$$

subject to the conditions (2), (3). Generally, the problem is well-posed. Using the solution of the problem we can calculate the quantity $u_{y}(x, 1)=\phi(x)$.

Problem 2: finding a function $u(x, y) \quad x \in \mathbb{R}, 0<y<1$ satisfying

$$
\Delta u=f, \quad x \in \mathbb{R}, 0<y<1
$$

subject to the conditions $u(x, 1)=\varphi(x), u_{y}(x, 1)=\phi(x)$.

The latter problem is a Cauchy elliptic problem and, as known, it is severely ill-posed. Hence, a regularization is in order.

The homogeneous problem $(f \equiv 0)$ was studied, by various methods in many papers. Using the mollification method, the homogeneous sideways parabolic problems were considered in [HRS, HR1, AS, L, LV, M] and the references therein. Similarly, the number of papers devoted to the Cauchy problem for linear homogeneous elliptic equation are very rich (see, e.g., [HR, T, B, CHWY, KS]).

Although there are many papers on homogeneous cases, we only find a few papers on nonhomogeneous sideways problems (for both parabolic and elliptic equations). Especially, the papers on the nonlinear case are very rare. In $[\mathrm{QT}]$, we use the method of integral equations to consider a sideways elliptic equation with a nonlinear heat source. However, we cannot get an effective method to regularize the problem. In the present paper, we shall consider the problem with a nonlinear heat source $f \equiv f(x, y, u(x, y))$. The remainder of our paper is divided in three sections. In Section 2, using Green functions, we shall transform Problems 1 and 2 into integral equations. In Section 3, we shall prove that Problem 1 has a unique solution. Moreover, we shall give an effective way to approximate the quantity $u_{y}(x, 1)$. In Section 4 , using $\varphi(x), u_{y}(x, 1)$, we shall regularize Problem 2 . The main result of the section and of the paper is Theorem 3 (in Subsection 4.2). The method of truncated high frequencies (of Fourier images) will be used and the regularized solution can be found as a fixed point of a contraction. In our knowledge, the latter method is new. Error estimates are given.

\section{Integral equations of Problems 1 and 2}

\subsection{Problem 1}


Put

$$
N(x, y, \xi, \eta)=-\frac{1}{4 \pi} \ln \frac{(x-\xi)^{2}+(y-\eta)^{2}}{(x-\xi)^{2}+(y+\eta-2)^{2}} .
$$

For $y>1, x \in \mathbb{R}$, integrating the identity

$$
\frac{\partial}{\partial \xi}\left(u N_{\xi}-N u_{\xi}\right)+\frac{\partial}{\partial \eta}\left(u N_{\eta}-N u_{\eta}\right)=-N f
$$

over the domain $(-m, m) \times(1, n) \backslash B((x, y), \varepsilon)$, where $B((x, y), \varepsilon)$ is the ball with center at $(x, y)$ and radius $\varepsilon>0$, and letting $n \rightarrow \infty, m \rightarrow \infty, \varepsilon \rightarrow 0$, we get, after some rearrangements,

$$
u(x, y)=A u(x, y)
$$

where

$$
A u(x, y)=h(x, y, \varphi)-\int_{-\infty}^{+\infty} \int_{1}^{+\infty} N(x, y ; \xi, \eta) f(\xi, \eta, u(\xi, \eta)) d \xi d \eta
$$

and

$$
h(x, y, \varphi)=\int_{-\infty}^{+\infty} N_{\eta}(x, y, \xi, 1) \varphi(\xi) d \xi
$$

\subsection{Problem 2}

We repeat $\psi(x)=u_{y}(x, 1)$ with $u$ being an exact solution of Problem 1 . We note that we only consider $\varphi$ as an exact data. The function $\psi$ is a processed data dependent on $\varphi$. In fact, in Lemma 1 (Section 3), we shall construct a function $\psi_{\varepsilon}$ which is an approximation of $\psi$.

Consider

$$
\Delta u=f(x, y, u(x, y)), \quad x \in \mathbb{R}, y \in(0,1)
$$

subject to the boundary conditions below

$$
\begin{gathered}
u(x, 1)=\varphi(x), \quad x \in \mathbb{R}, \\
\frac{\partial u}{\partial y}(x, 1)=\psi(x), \quad x \in \mathbb{R} .
\end{gathered}
$$

We assume in addition that the exact solution $u$ satisfying $\widehat{\varphi}(\zeta) e^{|\zeta|}, \widehat{\psi}(\zeta) e^{|\zeta|} \in L^{2}(\mathbb{R})$. We divide this problem into two problems.

Problem 2.1 Consider the problem

$$
\Delta v=0, \quad x \in \mathbb{R}, \quad y \in(0,1)
$$




$$
\begin{gathered}
v(x, 1)=\varphi(x), x \in \mathbb{R}, \\
\frac{\partial v}{\partial y}(x, 1)=\psi(x), x \in \mathbb{R} .
\end{gathered}
$$

We shall prove that, under appropriate conditions, the problem has a unique solution $v_{0}$ approximated by a regularized solution $v_{\varepsilon}$.

Problem 2.2 Let $u_{0}$ be an exact solution of (10). If we put $w_{0}=u_{0}-v_{0}$ then $w_{0}$ is the solution of the problem

$$
\begin{gathered}
\Delta w=g(x, y, w), \quad x \in \mathbb{R}, \quad y \in(0,1) \\
w(x, 1)=0, \quad x \in \mathbb{R}, \\
\frac{\partial w}{\partial y}(x, 1)=0, \quad x \in \mathbb{R}
\end{gathered}
$$

where $g(x, y, w)=f\left(x, y, w+v_{0}\right)$. We shall find a $w_{\varepsilon}$ which is an approximation of $w_{0}$ and estimate $\left\|w_{\varepsilon}-w_{0}\right\|_{2}$ where $\|\cdot\|_{2}$ is the norm in $L^{2}(\mathbb{R} \times(0,1))$. Let $u_{\varepsilon}=v_{\varepsilon}+w_{\varepsilon}$, we shall estimate $\left\|u_{\varepsilon}-u_{0}\right\|_{2}$.

\subsubsection{An integral equation of Problem 2.1}

Let

$$
\Gamma(x, y, \xi, \eta)=-\frac{1}{4 \pi} \ln \left[(x-\xi)^{2}+(y-\eta)^{2}\right]
$$

and

$$
G(x, y, \xi, \eta)=\Gamma(x, y, \xi, \eta)-\Gamma(x,-y, \xi, \eta)
$$

For $x \in \mathbb{R}, 0<y<1$, integrating the identity

$$
\frac{\partial}{\partial \xi}\left(-v G_{\xi}+G v_{\xi}\right)+\frac{\partial}{\partial \eta}\left(-v G_{\eta}+G v_{\eta}\right)=0
$$

over the domain $(-n, n) \times(0,1) \backslash B((x, y), \varepsilon)$ and letting $n \rightarrow \infty, \varepsilon \rightarrow 0$, we get, after some rearrangements

$$
\begin{aligned}
v(x, y)= & -\int_{-\infty}^{+\infty}\left[\varphi(\xi) G_{\eta}(x, y, \xi, 1)-G(x, y, \xi, 1) \psi(\xi)\right] d \xi+ \\
& +\int_{-\infty}^{+\infty} G_{\eta}(x, y, \xi, 0) v(\xi, 0) d \xi .
\end{aligned}
$$


Letting $y \rightarrow 1$ in (18), we have

$$
\frac{1}{\pi} \int_{-\infty}^{+\infty} \frac{1}{(x-\xi)^{2}+1} v(\xi, 0) d \xi+\int_{-\infty}^{+\infty}\left[-\varphi(\xi) G_{\eta}(x, 1, \xi, 1)+G(x, 1, \xi, 1) \psi(\xi)\right] d \xi=\varphi(x) .
$$

It can be rewritten as

$$
F_{(1)} * v_{(0)}(x)=\pi K_{(1)}(x)+\frac{\sqrt{\pi}}{\sqrt{2}} \varphi(x)
$$

where

$$
\begin{aligned}
& K_{(y)}(x)=-\frac{1}{\sqrt{2 \pi}} \int_{-\infty}^{+\infty}\left[-\varphi(\xi) G_{\eta}(x, y, \xi, 1)+G(x, y, \xi, 1) \psi(\xi)\right] d \xi \\
& F_{(y)}(x) \equiv \frac{y}{x^{2}+y^{2}}, v_{(y)}(x)=v(x, y)
\end{aligned}
$$

Letting

$$
M_{(y, 1)}(x)=\frac{1-y}{x^{2}+(y-1)^{2}}-\frac{1+y}{x^{2}+(y+1)^{2}}
$$

and

$$
L_{(\eta, y)}(x) \equiv \ln \frac{x^{2}+(y-\eta)^{2}}{x^{2}+(y+\eta)^{2}} \quad(0<y, \eta<1, x \in \mathbb{R}),
$$

we have the Fourier transform of $M, L, F$ as followed

$$
\begin{aligned}
\hat{F}_{(y)}(\zeta) & =\frac{1}{\sqrt{2 \pi}} \int_{-\infty}^{+\infty} F_{(y)}(x) e^{-i x \zeta} d x=\frac{\sqrt{\pi}}{\sqrt{2}} e^{-y|\zeta|}, \\
\hat{L}_{(\eta, y)}(\zeta) & =\sqrt{2 \pi} \frac{1}{|\zeta|}\left[e^{-(y+\eta)|\zeta|}-e^{-|y-\eta||\zeta|}\right], \\
\hat{M}_{(y, 1)}(\zeta) & =\frac{\sqrt{\pi}}{\sqrt{2}}\left[e^{(y-1)|\zeta|}-e^{-(y+1)|\zeta|}\right] .
\end{aligned}
$$

From (20), (21), we get

$$
K_{(y)}(x)=-\frac{1}{4 \pi}\left[2 \varphi * M_{(y, 1)}(x)-\psi * L_{(1, y)}(x)\right] .
$$

From (19), (20), we have

$$
\hat{v}_{(0)}(\zeta)=e^{|\zeta|}\left(\sqrt{2 \pi} \hat{K}_{(1)}(\zeta)+\hat{\varphi}(\zeta)\right)
$$


Taking the Fourier transform of (18), we get

$$
\begin{aligned}
\hat{v}_{(y)}(\zeta) & =e^{|\zeta|} \hat{F}_{(y)}(\zeta)\left(2 \hat{K}_{(1)}(\zeta)+\frac{\sqrt{2}}{\sqrt{\pi}} \hat{\varphi}(\zeta)\right)-\sqrt{2 \pi} \hat{K}_{(y)}(\zeta) \\
& =\frac{1}{2} \hat{\varphi}(\zeta)\left[e^{(1-y)|\zeta|}+e^{(y-1)|\zeta|}\right]-\frac{1}{2|\zeta|} \widehat{\psi}(\zeta)\left[e^{(1-y)|\zeta|}-e^{(y-1)|\zeta|}\right] \equiv \aleph(\zeta, y) .
\end{aligned}
$$

\subsubsection{An integral equation of Problem 2.2}

We recall that $w_{0}$ an exact solution of Problem (14) - (16).

Let $v_{0} \in L^{2}(\mathbb{R} \times(0,1))$ be the exact solution of (11) - (13) and $v_{\varepsilon} \in L^{2}(\mathbb{R} \times(0,1))$ be a regularized solution.

We write $w_{(y)}(x)=w(x, y)$ and $f_{\left(\eta, w_{0}, v_{0}\right)}(\xi)=f\left(\xi, \eta, v_{0}(\xi, \eta)+w_{0}(\xi, \eta)\right)$.

For $x \in \mathbb{R}, 0<y<1$, let $G$ be defined in (17), integrating the identity

$$
\frac{\partial}{\partial \xi}\left(-w_{0} G_{\xi}+G w_{0 \xi}\right)+\frac{\partial}{\partial \eta}\left(-w_{0} G_{\eta}+G w_{0 \eta}\right)=G f_{\left(\eta, w_{0}, v_{0}\right)}(\xi)
$$

over the domain $(-n, n) \times(0,1) \backslash B((x, y), \varepsilon)$ and letting $n \rightarrow \infty, \varepsilon \rightarrow 0$, we get, after some rearrangements,

$$
w_{0}(x, y)=\int_{-\infty}^{+\infty} w_{0}(\xi, 0) G_{\eta}(x, y, \xi, 0) d \xi-\int_{-\infty}^{+\infty} \int_{0}^{1} G(x, y, \xi, \eta) f_{\left(\eta, w_{0}, v_{0}\right)}(\xi) d \xi d \eta
$$

From (26), we have

$$
\begin{aligned}
w_{0}(x, y)= & \frac{1}{\pi} \int_{-\infty}^{+\infty} w_{0}(\xi, 0) \frac{y}{(x-\xi)^{2}+y^{2}} d \xi \\
& +\frac{1}{4 \pi} \int_{0}^{1} \int_{-\infty}^{+\infty} \ln \frac{(x-\xi)^{2}+(y-\eta)^{2}}{(x-\xi)^{2}+(y+\eta)^{2}} f_{\left(\eta, w_{0}, v_{0}\right)}(\xi) d \xi d \eta .
\end{aligned}
$$

Letting $y \rightarrow 1$, we get

$$
\frac{1}{4} \int_{0}^{1} \int_{-\infty}^{+\infty} \ln \frac{(x-\xi)^{2}+(1-\eta)^{2}}{(x-\xi)^{2}+(1+\eta)^{2}} f_{\left(\eta, w_{0}, v_{0}\right)}(\xi) d \xi d \eta+\int_{-\infty}^{+\infty} w_{0}(\xi, 0) \frac{1}{(x-\xi)^{2}+1} d \xi=0 .
$$

From (21), we get

$$
\begin{aligned}
& F_{(y)}(x) \equiv \frac{y}{x^{2}+y^{2}}, L_{(\eta, y)}(x) \equiv \ln \frac{x^{2}+(y-\eta)^{2}}{x^{2}+(y+\eta)^{2}} \quad(0<y, \eta<1, x \in \mathbb{R}), \\
& \hat{F}_{(y)}(\zeta)=\frac{\sqrt{\pi}}{\sqrt{2}} e^{-y|\zeta|}, \hat{L}_{(\eta, y)}(\zeta)=\sqrt{2 \pi} \frac{1}{|\zeta|}\left[e^{-(y+\eta)|\zeta|}-e^{-|y-\eta||\zeta|}\right] .
\end{aligned}
$$


We write

$$
w_{0(y)}(x) \equiv w_{0}(x, y) .
$$

From (28), (29) can be rewritten as

$$
w_{0(0)} * F_{(1)}(x)+\frac{1}{4} \int_{0}^{1} L_{(\eta, 1)} * f_{\left(\eta, w_{0}, v_{0}\right)}(x) d \eta=0
$$

Taking the Fourier transform, we have

$$
\widehat{w_{0(0)}}(\zeta) \cdot \hat{F}_{(1)}(\zeta)+\frac{1}{4} \int_{0}^{1} \hat{L}_{(\eta, 1)}(\zeta) \hat{f}_{\left(\eta, w_{0}, v_{0}\right)}(\zeta) d \eta=0 .
$$

From (29), we have

$$
\widehat{w_{0(0)}}(\zeta)=-\frac{1}{2} \int_{0}^{1} \frac{1}{|\zeta|}\left[e^{-\eta|\zeta|}-e^{\eta|\zeta|}\right] \hat{f}_{\left(\eta, w_{0}, v_{0}\right)}(\zeta) d \eta
$$

From (26), we have

$$
w_{0(y)}(x)=\frac{\sqrt{2}}{\sqrt{\pi}} w_{0(0)} * F_{(y)}(x)+\frac{1}{2 \sqrt{2 \pi}} \int_{0}^{1} L_{(\eta, y)} * f_{\left(\eta, w_{0}, v_{0}\right)}(x) d \eta .
$$

Taking the Fourier transform of (31), we get

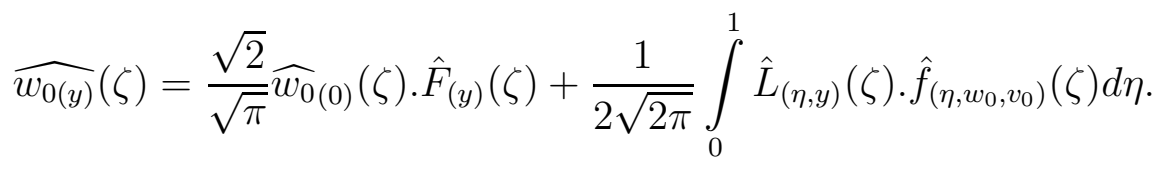

From (29) and (30), Eq. (32) takes the form

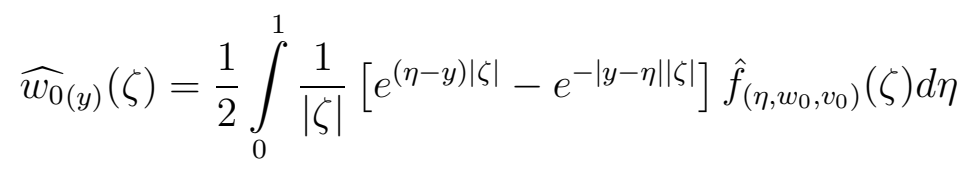

for $\zeta$. We have

$$
{\widehat{w_{0}}}_{(y)}(\zeta)=\frac{1}{2 \sqrt{2 \pi}} \int_{0}^{1} \int_{-\infty}^{+\infty} \frac{1}{|\zeta|}\left[e^{(\eta-y)|\zeta|}-e^{-|y-\eta||\zeta|}\right] f_{\left(\eta, w_{0}, v_{0}\right)}(\xi) e^{-i \xi \zeta} d \xi d \eta
$$




\section{Finding the solution of Problem 1}

From (8)-(9), we readily get the following result

Theorem 1. (see $[Q T])$ Suppose that for all $(\xi, \eta, \zeta) \in \mathbb{R} \times \mathbb{R}^{+} \times \mathbb{R}$

$$
\left|f_{\zeta}^{\prime}(\xi, \eta, \zeta)\right| \leq p(\xi, \eta)
$$

where $p(\xi, \eta) \in L^{1}(\mathbb{R} \times(1,+\infty)), p \geq 0$ satisfies

$$
K \equiv \sup _{(x, y) \in \mathbb{R} \times(1,+\infty)}\left|\int_{-\infty}^{+\infty} \int_{1}^{+\infty} N(x, y ; \xi, \eta) p(\xi, \eta) d \xi d \eta\right|<1 .
$$

Put

$$
J=\left\{u \in C(\mathbb{R} \times(1,+\infty)) \mid \lim _{\sqrt{x^{2}+y^{2}} \rightarrow+\infty} u(x, y)=0\right\} .
$$

Then $A: J \rightarrow J$ is a contraction and hence $u$ is uniquely determined and can be found by successive approximation.

Let $\psi(x)=u_{y}(x, 1)$ with $u$ be an exact solution in the half plane $x \in \mathbb{R}, y>1$. From a measured data $\varphi_{\varepsilon}$ of $\varphi(x)=u(x, 1)$, we construct $\psi_{\varepsilon}$ being an aproximation of $\psi$ and estimate $\left\|\psi_{\varepsilon}-\psi\right\|_{L^{2}(\mathbb{R})}$ by the following lemma

\section{Lemma 1}

Let $u$ be a solution of (8) as in Theorem 1 and let $\varphi \in L^{1}(\mathbb{R}) \cap L^{\infty}(\mathbb{R})$ satisfy $\hat{\varphi}(\zeta) e^{|\zeta|} \in L^{2}(\mathbb{R})$.

Suppose that $f$ satisfies the conditions

$$
\left|f\left(\xi, \eta, \zeta_{1}\right)-f\left(\xi, \eta, \zeta_{2}\right)\right| \leq|p(\xi, \eta)|\left|\zeta_{1}-\zeta_{2}\right| \quad \text { for all }(\xi, \eta) \in \mathbb{R} \times \mathbb{R}^{+}, \zeta_{1}, \zeta_{2} \in \mathbb{R}
$$

where $p \in L^{1}(\mathbb{R} \times(1,+\infty))$,

$$
K \equiv \sup _{(x, y) \in \mathbb{R} \times(1,+\infty)} \int_{-\infty}^{+\infty} \int_{1}^{+\infty} N(x, y ; \xi, \eta)|p(\xi, \eta)| d \xi d \eta<1
$$

and

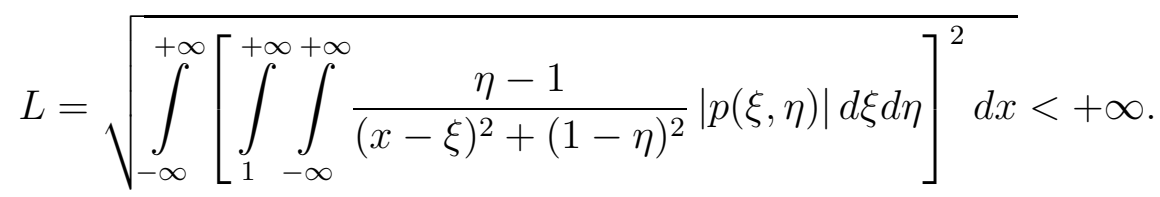

For every $0<\varepsilon<1$, we call $\varphi_{\varepsilon} \in L^{2}(\mathbb{R})$ a measured data such that

$$
\left\|\varphi_{\varepsilon}-\varphi\right\|_{L^{2}(\mathbb{R})}<\varepsilon
$$


From $\varphi_{\varepsilon}$, we can construct a function $\psi_{\varepsilon} \in L^{2}(\mathbb{R})$ such that

$$
\left\|\psi_{\varepsilon}-\psi\right\|_{L^{2}(\mathbb{R})}<C \varepsilon^{1 / 2}
$$

where $C$ is independent on $\varepsilon$.

\section{Proof}

Let

$$
k(x, y, u)=-\int_{-\infty}^{+\infty} \int_{1}^{+\infty} N(x, y ; \xi, \eta) f(\xi, \eta, u(\xi, \eta)) d \xi d \eta
$$

and

$$
h(x, y, \varphi)=\int_{-\infty}^{+\infty} N_{\eta}(x, y, \xi, 1) \varphi(\xi) d \xi .
$$

We have $u(x, y)=h(x, y, \varphi)+k(x, y, u)$.

Put

$$
\widetilde{\varphi_{\varepsilon}}(x)=\frac{1}{\sqrt{2 \pi}} \int_{|\zeta|<\frac{1}{\varepsilon^{1 / 2}}} \widehat{\varphi_{\varepsilon}}(\zeta) e^{i \zeta x} d \zeta .
$$

We have

$$
\widehat{\varphi_{\varepsilon}}(\zeta)= \begin{cases}\widehat{\varphi_{\varepsilon}}(\zeta) & |\zeta|<\frac{1}{\varepsilon^{1 / 2}} \\ 0 & |\zeta| \geq \frac{1}{\varepsilon^{1 / 2}}\end{cases}
$$

Let $u_{\varepsilon}$ be a solution of (8) with $\varphi$ replaced by $\widetilde{\varphi_{\varepsilon}}$, i.e.

$$
u_{\varepsilon}=h\left(x, y, \widetilde{\varphi_{\varepsilon}}\right)+k\left(x, y, u_{\varepsilon}\right) .
$$

We denote

$$
\begin{aligned}
h(x, y, \varphi) & =h(x, y), \\
h\left(x, y, \widetilde{\varphi_{\varepsilon}}\right) & =h_{\varepsilon}(x, y), \\
k(x, y, u) & =k(x, y), \\
k\left(x, y, u_{\varepsilon}\right) & =k_{\varepsilon}(x, y) .
\end{aligned}
$$

Let

$$
\psi_{\varepsilon}(x)=h_{\varepsilon y}(x, 1)+k_{\varepsilon y}(x, 1) .
$$


We have

$$
\begin{aligned}
h(x, y) & =\int_{-\infty}^{+\infty} N_{\eta}(x, y ; \xi, 1) \varphi(\xi) d \xi \\
& =\frac{1}{\pi} \int_{-\infty}^{+\infty} \frac{y-1}{(x-\xi)^{2}+(y-1)^{2}} \varphi(\xi) d \xi
\end{aligned}
$$

If we put $F_{(y)}(x) \equiv \frac{y}{x^{2}+y^{2}}, y>0$, then

$$
\hat{F}_{(y)}(\zeta)=\frac{1}{\sqrt{2 \pi}} \int_{-\infty}^{+\infty} F_{(y)}(x) e^{-i x \zeta} d x=\frac{\sqrt{\pi}}{\sqrt{2}} e^{-y|\zeta|}
$$

Taking the Fourier transform of (42), we get

$$
\widehat{h}(\zeta, y)=\frac{\sqrt{2}}{\sqrt{\pi}} \widehat{F(y-1)} * \varphi(\zeta)=\widehat{\varphi}(\zeta) e^{-(y-1)|\zeta|}
$$

and

$$
\widehat{h}_{y}(\zeta, y)=-|\zeta| \widehat{\varphi}(\zeta) e^{-(y-1)|\zeta|}
$$

Similarly, we have

$$
\widehat{h_{\varepsilon}}(\zeta, y)=\widehat{\widetilde{\varphi}}(\zeta) e^{-(y-1)|\zeta|}
$$

We shall find an estimation of $\left\|h_{\varepsilon y}(., 1)-h_{y}(., 1)\right\|_{L^{2}(\mathbb{R})}$.

Using the inequality $u^{4}<e^{2 u} \quad \forall u>1$, we have

$$
\begin{aligned}
\left\|h_{\varepsilon y}(., 1)-h_{y}(., 1)\right\|_{L^{2}(\mathbb{R})}^{2} & =\int_{-\infty}^{+\infty}|\zeta|^{2}\left|\widehat{\varphi}(\zeta)-\widehat{\varphi_{\varepsilon}}(\zeta)\right|^{2} d \zeta \\
& \leq \int_{|\zeta|<\frac{1}{\varepsilon^{1 / 2}}}|\zeta|^{2}\left|\widehat{\varphi}(\zeta)-\widehat{\varphi_{\varepsilon}}(\zeta)\right|^{2} d \zeta+\int_{|\zeta|>\frac{1}{\varepsilon^{1 / 2}}} \frac{e^{2|\zeta|}}{|\zeta|^{2}}|\widehat{\varphi}(\zeta)|^{2} d \zeta .
\end{aligned}
$$

Therefore

$$
\left\|h_{\varepsilon y}(., 1)-h_{y}(., 1)\right\|_{L^{2}(\mathbb{R})}^{2}<\varepsilon+\varepsilon\left\|e^{|\zeta|} \widehat{\varphi}(\zeta)\right\|_{L^{2}(\mathbb{R})}^{2}=C_{1}^{2} \varepsilon
$$

where $C_{1}=\sqrt{1+\left\|e^{|\zeta|} \widehat{\varphi}(\zeta)\right\|_{L^{2}(\mathbb{R})}^{2}}$.

Hence

$$
\left\|h_{\varepsilon y}(., 1)-h_{y}(., 1)\right\|_{L^{2}(\mathbb{R})}<C_{1} \sqrt{\varepsilon}
$$


We have

$$
k(x, y)=-\int_{1}^{+\infty} \int_{-\infty}^{+\infty} N(x, y ; \xi, \eta) f(\xi, \eta, u(\xi, \eta)) d \xi d \eta
$$

It follows that

$$
k_{y}(x, 1)=\frac{1}{\pi} \int_{1}^{+\infty} \int_{-\infty}^{+\infty} \frac{1-\eta}{(x-\xi)^{2}+(1-\eta)^{2}} f(\xi, \eta, u(\xi, \eta)) d \xi d \eta
$$

Similarly, we get

$$
k_{\varepsilon y}(x, 1)=\frac{1}{\pi} \int_{1}^{+\infty} \int_{-\infty}^{+\infty} \frac{1-\eta}{(x-\xi)^{2}+(1-\eta)^{2}} f\left(\xi, \eta, u_{\varepsilon}(\xi, \eta)\right) d \xi d \eta .
$$

We have

$$
\begin{aligned}
& \left\|k_{\varepsilon y}(., 1)-k_{y}(., 1)\right\|_{L^{2}(\mathbb{R})}= \\
& =\frac{1}{\pi} \sqrt{\int_{-\infty}^{+\infty}\left(\int_{1}^{+\infty} \int_{-\infty}^{+\infty} \frac{1-\eta}{(x-\xi)^{2}+(1-\eta)^{2}}\left(f(\xi, \eta, u(\xi, \eta))-f\left(\xi, \eta, u_{\varepsilon}(\xi, \eta)\right) d \xi d \eta\right)^{2} d x\right.} \\
& \leq \frac{1}{\pi} \sqrt{\int_{-\infty}^{+\infty}\left[\int_{1}^{+\infty} \int_{-\infty}^{+\infty} \frac{\eta-1}{(x-\xi)^{2}+(1-\eta)^{2}}|p(\xi, \eta)|\left|u-u_{\varepsilon}\right| d \xi d \eta\right]^{2}} d x \\
& \leq \frac{1}{\pi}\left\|u-u_{\varepsilon}\right\|_{\infty} L
\end{aligned}
$$

where

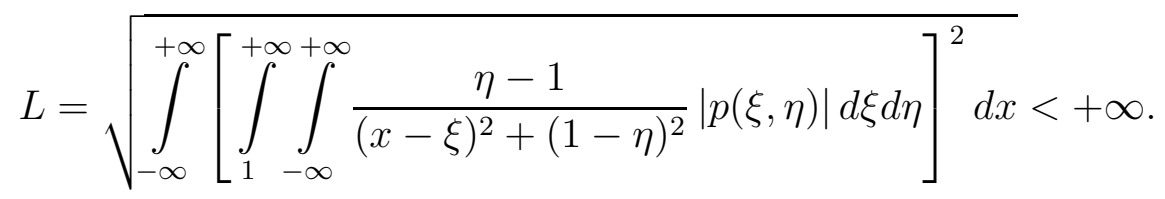

Moreover, we have

$$
\begin{aligned}
\left\|u-u_{\varepsilon}\right\|_{\infty} \leq & \sup _{(x, y) \in \mathbb{R} \times(1,+\infty)} \mid \int_{-\infty}^{+\infty} N_{\eta}(x, y, \xi, 1)\left[\varphi(\xi)-\widetilde{\varphi_{\varepsilon}}(\xi)\right] d \xi \\
& -\int_{-\infty}^{+\infty} \int_{1}^{+\infty} N(x, y, \xi, \eta)\left(f(\xi, \eta, u(\xi, \eta))-f\left(\xi, \eta, u_{\varepsilon}(\xi, \eta)\right)\right) d \xi d \eta \mid
\end{aligned}
$$




$$
\begin{aligned}
& \leq \sup _{(x, y) \in \mathbb{R} \times(1+\infty)}\left[\int_{-\infty}^{+\infty} \frac{1}{\pi} \frac{y-1}{(x-\xi)^{2}+(y-1)^{2}}\left|\varphi(\xi)-\widetilde{\varphi}_{\varepsilon}(\xi)\right| d \xi\right. \\
& \left.\quad+\int_{-\infty}^{+\infty} \int_{1}^{+\infty} N(x, y, \xi, \eta)|p(\xi, \eta)|\left|u(\xi, \eta)-u_{\varepsilon}(\xi, \eta)\right| d \xi d \eta\right] \\
& \leq\left\|\varphi-\widetilde{\varphi_{\varepsilon}}\right\|_{L^{\infty}(\mathbb{R})}+K\left\|u-u_{\varepsilon}\right\|_{\infty}
\end{aligned}
$$

where

$$
K=\sup _{(x, y) \in \mathbb{R} \times(1+\infty)} \int_{-\infty}^{+\infty} \int_{1}^{+\infty} N(x, y, \xi, \eta)|p(\xi, \eta)| d \xi d \eta \in(0,1)
$$

Hence

$$
\left\|u-u_{\varepsilon}\right\|_{\infty} \leq \frac{1}{1-K}\left\|\varphi-\widetilde{\varphi_{\varepsilon}}\right\|_{L^{\infty}(\mathbb{R})} .
$$

We get

$$
\begin{aligned}
\left\|\varphi-\widetilde{\varphi_{\varepsilon}}\right\|_{L^{\infty}(\mathbb{R})} & =\sup _{x \in \mathbb{R}}\left|\widetilde{\varphi_{\varepsilon}}(x)-\varphi(x)\right| \\
& =\sup _{x \in \mathbb{R}} \frac{1}{\sqrt{2 \pi}}\left|\int_{|\zeta|<\frac{1}{\varepsilon^{1 / 2}}} \widehat{\varphi_{\varepsilon}}(\zeta) e^{i \zeta x} d \zeta-\int_{-\infty}^{+\infty} \widehat{\varphi}(\zeta) e^{i \zeta x} d \zeta\right| \\
\leq & \frac{1}{\sqrt{2 \pi}}\left[\int_{\left.|\zeta|<\frac{1}{\varepsilon^{1 / 2}}\left|\widehat{\varphi_{\varepsilon}}(\zeta)-\widehat{\varphi}(\zeta)\right| d \zeta+\int_{|\zeta|>\frac{1}{\varepsilon^{1 / 2}}}|\widehat{\varphi}(\zeta)| d \zeta\right]}\right. \\
\leq & \frac{1}{\sqrt{2 \pi}}\left[\sqrt{\int_{-\frac{1}{\varepsilon^{1 / 2}}}^{\frac{1}{\varepsilon^{1 / 2}}} d \zeta \int_{\frac{1}{\varepsilon^{1 / 2}}}^{\frac{1}{\varepsilon^{1 / 2}}}\left|\widehat{\varphi_{\varepsilon}}(\zeta)-\widehat{\varphi}(\zeta)\right|^{2} d \zeta+}\right. \\
& \left.+\sqrt{|\zeta|>\frac{1}{\varepsilon^{1 / 2}}} e^{2|\zeta|}|\widehat{\varphi}(\zeta)|^{2} d \zeta . \int_{|\zeta|>\frac{1}{\varepsilon^{1 / 2}}} e^{-2|\zeta|} d \zeta\right] \\
\leq & \frac{1}{\sqrt{2 \pi}}\left[\frac{\sqrt{2}}{\varepsilon^{1 / 4}}\left\|\varphi-\varphi_{\varepsilon}\right\|_{L^{2}(\mathbb{R})}+\sqrt{\frac{2}{3}}\left\|e^{|\zeta|}(\zeta)\right\|_{L^{2}(\mathbb{R})} \varepsilon^{3 / 4}\right] \\
< & C_{2} \varepsilon^{3 / 4}
\end{aligned}
$$


where

$$
C_{2}=\frac{1}{\sqrt{2 \pi}}\left[\sqrt{2}+\sqrt{\frac{2}{3}}\left\|e^{|\zeta|} \widehat{\varphi}(\zeta)\right\|_{L^{2}(\mathbb{R})}\right] .
$$

Inequalities (44) - (47) give

$$
\left\|k_{\varepsilon y}(., 1)-k_{y}(., 1)\right\|_{L^{2}(\mathbb{R})} \leq \frac{L}{\pi(1-K)}\left\|\varphi-\widetilde{\varphi}_{\varepsilon}\right\|_{\infty}<\frac{L C_{2}}{\pi(1-K)} \varepsilon^{3 / 4} .
$$

In view of (38) - (41), (43) and (48), we have

$$
\begin{aligned}
\left\|\psi_{\varepsilon}-\psi\right\|_{L^{2}(\mathbb{R})} & =\left\|u_{\varepsilon y}(., 1)-u_{y}(., 1)\right\|_{L^{2}(\mathbb{R})} \\
& =\left\|h_{\varepsilon y}(., 1)+k_{\varepsilon y}(., 1)-h_{y}(., 1)-k_{y}(., 1)\right\|_{L^{2}(\mathbb{R})} \\
& \leq\left\|h_{\varepsilon y}(., 1)-h_{y}(., 1)\right\|_{L^{2}(\mathbb{R})}+\left\|k_{\varepsilon y}(., 1)-k_{y}(., 1)\right\|_{L^{2}(\mathbb{R})} \\
& <\frac{L C_{2}}{\pi(1-K)} \varepsilon^{3 / 4}+C_{1} \varepsilon^{1 / 2} \leq\left(\frac{L C_{2}}{\pi(1-K)}+C_{1}\right) \varepsilon^{1 / 2} .
\end{aligned}
$$

This complete the proof of Lemma 1.

\section{Regularization of problem}

We shall first regularize the Problem 2.1 (Subsection 4.1). Using the approximated solution $v_{\varepsilon}$ of Problem 2.1, we shall regularize the Problem 2.2 (Subsection 4.2).

\subsection{Problem 2.1 (Problem (24))}

We have the following result

\section{Theorem 1}

Let $\varphi \in L^{1}(\mathbb{R}) \cap L^{\infty}(\mathbb{R})$. Suppose $\widehat{\varphi}(\zeta) e^{|\zeta|} \in L^{2}(\mathbb{R}), \widehat{\psi}(\zeta) e^{|\zeta|} \in L^{2}(\mathbb{R})$. Then Problem (11) - (13) has a unique solution $v_{0} \in L^{2}(\mathbb{R} \times(0,1))$.

Proof

From (24) and the inequality $\frac{e^{|x|}-1}{|x|} \leq e^{|x|}$ we have

$$
|\aleph(\zeta, y)| \leq e^{|\zeta|}|\widehat{\varphi}(\zeta)|+e^{|\zeta|}|\widehat{\psi}(\zeta)| \in L^{2}(\mathbb{R}) \text {, for all } 0 \leq y \leq 1
$$

where $\aleph$ is as in (24).

Hence Problem (11) - (13) has a (unique) solution $v_{0} \in L^{2}(\mathbb{R} \times(0,1))$.

The proof is completed.

\section{Theorem 2}

Let assumptions in Theorem 1 hold.

For every $0<\varepsilon<e^{-3}$, let $\varphi_{\varepsilon} \in L^{2}(\mathbb{R})$ be measured data such that $\left\|\varphi_{\varepsilon}-\varphi\right\|_{L^{2}(\mathbb{R})}<\varepsilon$.

If, in addition, the assumptions of Lemma 1 hold and we have $|\zeta| \widehat{\varphi}(\zeta) e^{|\zeta|} \in L^{2}(\mathbb{R})$, $|\zeta| \widehat{\psi}(\zeta) e^{|\zeta|} \in L^{2}(\mathbb{R})$. 
Then, from $\varphi_{\varepsilon}$, we can construct a regularized solution $v_{\varepsilon}$ such that $\left\|v_{0}-v_{\varepsilon}\right\|_{2}<D\left(\ln \frac{1}{\varepsilon}\right)^{-1}$, where $\|\cdot\|_{2}$ is the norm in $L^{2}(\mathbb{R} \times(0,1))$ and $D$ is a positive constant independent of $\varepsilon$.

Proof

We recall that $\widetilde{\varphi_{\varepsilon}}$ is defined in Lemma 1 , we get

$$
\begin{aligned}
\left\|\widetilde{\varphi_{\varepsilon}}-\varphi\right\|_{L^{2}(\mathbb{R})}^{2} & =\int_{|\zeta|<\varepsilon^{-1 / 2}}\left|\widehat{\varphi_{\varepsilon}}(\zeta)-\widehat{\varphi}(\zeta)\right|^{2} d \zeta+\int_{|\zeta|>\varepsilon^{-1 / 2}}|\widehat{\varphi}(\zeta)|^{2} d \zeta \\
& <\varepsilon^{2}+\int_{|\zeta|>\varepsilon^{-1 / 2}} \frac{|\widehat{\varphi}(\zeta)|^{2} e^{2|\zeta|}}{|\zeta|^{4}} d \zeta \\
& <\varepsilon^{2}+\varepsilon^{2}\left\|\widehat{\varphi}(\zeta) e^{|\zeta|}\right\|_{L^{2}(\mathbb{R})}^{2}=\varepsilon^{2} C_{3}^{2}
\end{aligned}
$$

where $C_{3}=\sqrt{1+\left\|\widehat{\varphi}(\zeta) e^{|\zeta|}\right\|_{L^{2}(\mathbb{R})}^{2}}$.

Therefore $\left\|\widetilde{\varphi_{\varepsilon}}-\varphi\right\|_{L^{2}(\mathbb{R})}<C_{3} \varepsilon$.

Put

$$
\phi_{\varepsilon}(x)=\frac{1}{\sqrt{2 \pi}} \int_{-\frac{1}{6} \ln \frac{1}{\varepsilon}}^{\frac{1}{6} \ln \frac{1}{\varepsilon}} \widehat{\widetilde{\varphi}}(\zeta) e^{i \zeta x} d \zeta
$$

We have

$$
\widehat{\phi}_{\varepsilon}(\zeta)= \begin{cases}\widehat{\varphi_{\varepsilon}}(\zeta) & |\zeta|<\frac{1}{6} \ln \frac{1}{\varepsilon} \\ 0 & |\zeta| \geq \frac{1}{6} \ln \frac{1}{\varepsilon}\end{cases}
$$

It follows that

$$
\begin{aligned}
\left\|\left(\widehat{\phi_{\varepsilon}}(\zeta)-\widehat{\varphi}(\zeta)\right) e^{|\zeta|}\right\|_{L^{2}(\mathbb{R})}^{2} & =\int_{-\frac{1}{6} \ln \frac{1}{\varepsilon}}\left|\widehat{\varphi_{\varepsilon}}(\zeta)-\widehat{\varphi}(\zeta)\right|^{2} e^{2|\zeta|} d \zeta+\int_{|\zeta|>\frac{1}{6} \ln \frac{1}{\varepsilon}}|\widehat{\varphi}(\zeta)|^{2} e^{2|\zeta|} d \zeta \\
& \leq e^{\frac{1}{3} \ln \frac{1}{\varepsilon}}\left\|\widetilde{\varphi_{\varepsilon}}-\varphi\right\|_{L^{2}(\mathbb{R})}^{2}+\frac{1}{\left(\frac{1}{6} \ln \frac{1}{\varepsilon}\right)^{2}}\left\|\zeta \widehat{\varphi}(\zeta) e^{|\zeta|}\right\|_{L^{2}(\mathbb{R})}^{2} \\
& <C_{3}^{2} e^{\frac{1}{3} \ln \frac{1}{\varepsilon} \varepsilon^{2}}+\frac{1}{\left(\frac{1}{6} \ln \frac{1}{\varepsilon}\right)^{2}}\left\|\zeta \widehat{\varphi}(\zeta) e^{|\zeta|}\right\|_{L^{2}(\mathbb{R})}^{2} \\
& <C_{3}^{2} \varepsilon^{5 / 3}+\frac{36}{\ln ^{2} \frac{1}{\varepsilon}}\left\|\zeta \widehat{\varphi}(\zeta) e^{|\zeta|}\right\|_{L^{2}(\mathbb{R})}^{2}
\end{aligned}
$$

Using the inequality $\varepsilon^{5 / 3}<\frac{1}{\ln ^{2} \frac{1}{\varepsilon}}$ as $\varepsilon<e^{-3}$, we get

$$
\left\|\left(\widehat{\phi}_{\varepsilon}(\zeta)-\widehat{\varphi}(\zeta)\right) e^{|\zeta|}\right\|_{L^{2}(\mathbb{R})}^{2}<C_{4}^{2} \frac{1}{\ln ^{2} \frac{1}{\varepsilon}}
$$


where

$$
C_{4}=\sqrt{C_{3}^{2}+36\left\|\zeta \widehat{\varphi}(\zeta) e^{|\zeta|}\right\|_{L^{2}(\mathbb{R})}^{2}}
$$

Using lemma 1 , there exists $\psi_{\varepsilon} \in L^{2}(\mathbb{R})$ such that

$$
\left\|\psi_{\varepsilon}-\psi\right\|_{L^{2}(\mathbb{R})}<C \varepsilon^{1 / 2} .
$$

Put

$$
\Psi_{\varepsilon}(x)=\frac{1}{\sqrt{2 \pi}} \int_{-\frac{1}{6} \ln \frac{1}{\varepsilon}}^{\frac{1}{6} \ln \frac{1}{\varepsilon}} \widehat{\Psi_{\varepsilon}}(\zeta) e^{i \zeta x} d \zeta
$$

We have

$$
\widehat{\Psi_{\varepsilon}}(\zeta)=\left\{\begin{array}{ll}
\widehat{\psi_{\varepsilon}}(\zeta) & |\zeta|<\frac{1}{6} \ln \frac{1}{\varepsilon} \\
0 & |\zeta| \geq \frac{1}{6} \ln \frac{1}{\varepsilon}
\end{array} .\right.
$$

Similarly, using the inequality $\varepsilon^{2 / 3}<\frac{1}{\ln ^{2} \frac{1}{\varepsilon}}$ as $\varepsilon<e^{-3}$, we get

$$
\left\|\left(\widehat{\Psi_{\varepsilon}}(\zeta)-\widehat{\psi}(\zeta)\right) e^{|\zeta|}\right\|_{L^{2}(\mathbb{R})}^{2}<C_{5}^{2} \frac{1}{\ln ^{2} \frac{1}{\varepsilon}}
$$

where

$$
C_{5}=\sqrt{C^{2}+36\left\|\zeta \widehat{\psi}(\zeta) e^{|\zeta|}\right\|_{L^{2}(\mathbb{R})}^{2}}
$$

We put

$$
\aleph_{\varepsilon}(\zeta, y)=\frac{1}{2} \widehat{\phi}_{\varepsilon}(\zeta)\left[e^{(1-y)|\zeta|}+e^{(y-1)|\zeta|}\right]-\frac{1}{2|\zeta|} \widehat{\Psi_{\varepsilon}}(\zeta)\left[e^{(1-y)|\zeta|}-e^{(y-1)|\zeta|}\right]
$$

and

$$
v_{\varepsilon}(x, y)=\frac{1}{\sqrt{2 \pi}} \int_{-\infty}^{+\infty} \aleph_{\varepsilon}(\zeta, y) e^{i \zeta x} d \zeta .
$$

From (24), (51), we get

$$
\begin{aligned}
& \left\|v_{\varepsilon}-v_{0}\right\|_{2}=\left\|\aleph_{\varepsilon}-\aleph\right\|_{2} \\
& \leq \frac{1}{2}\left(\left\|\left(\widehat{\varphi}(\zeta)-\widehat{\phi_{\varepsilon}}(\zeta)\right)\left[e^{(1-y)|\zeta|}+e^{(y-1)|\zeta|}\right]\right\|_{2}+\left\|\frac{\left(\widehat{\psi}(\zeta)-\widehat{\Psi_{\varepsilon}}(\zeta)\right)}{|\zeta|}\left[e^{(1-y)|\zeta|}-e^{(y-1)|\zeta|}\right]\right\|_{2}\right) \\
& \leq\left\|\left(\widehat{\varphi}(\zeta)-\widehat{\phi}_{\varepsilon}(\zeta)\right) e^{|\zeta|}\right\|_{2}+\left\|\left(\widehat{\psi}(\zeta)-\widehat{\Psi_{\varepsilon}}(\zeta)\right) e^{|\zeta|}\right\|_{2} \\
& \quad<\left(C_{4}+C_{5}\right)\left(\ln \frac{1}{\varepsilon}\right)^{-1} .
\end{aligned}
$$


Hence $\left\|v_{0}-v_{\varepsilon}\right\|_{2}<D\left(\ln \frac{1}{\varepsilon}\right)^{-1}$.

The proof is completed.

\subsection{Problem 2.2 (Problem (34))}

\section{Theorem 3}

Let assumptions in Theorem 1 and Theorem 2 hold.

Let $v_{0} \in L^{2}(\mathbb{R} \times(0,1))$ be an exact solution of (111) - (13) and $v_{\varepsilon} \in L^{2}(\mathbb{R} \times(0,1))$ be the regularized solution of (11) $-(13)$.

Suppose that $f$ satisfies the conditions (27) and $|p(\xi, \eta)| \leq k \forall(\xi, \eta) \in \mathbb{R} \times(0,1)$.

Assume in addition that the exact solution $u_{0}=v_{0}+w_{0} \in L^{2}(\mathbb{R} \times(0,1))$ of (10) satisfying

$$
e^{3|\zeta|}\left|\widehat{f}_{\left(\eta, v_{0}, w_{0}\right)}(\zeta)\right| \in L^{2}(\mathbb{R} \times(0,1)) .
$$

Then there exists a regularized solutions $w_{\varepsilon}$ and $u_{\varepsilon}=v_{\varepsilon}+w_{\varepsilon}$ of (10) such that

$$
\left\|w_{\varepsilon}-w_{0}\right\|_{2}<C\left(\ln \frac{1}{\varepsilon}\right)^{-1 / 2}
$$

and

$$
\left\|u_{\varepsilon}-u_{0}\right\|_{2}<E\left(\ln \frac{1}{\varepsilon}\right)^{-1 / 2}
$$

where $C$ and $E$ independent of $\varepsilon$.

Proof

From (33), we have

$$
\begin{aligned}
\widehat{w}_{0(y)}(\zeta) & =\frac{1}{2} \int_{0}^{1} \frac{1}{|\zeta|}\left[e^{(\eta-y)|\zeta|}-e^{-|y-\eta||\zeta|}\right] \widehat{f}_{\left(\eta, w_{0}, v_{0}\right)}(\zeta) d \eta \\
& =\frac{1}{2} \int_{y}^{1} \frac{1}{|\zeta|}\left[e^{(\eta-y)|\zeta|}-e^{(y-\eta)|\zeta|}\right] \widehat{f}_{\left(\eta, w_{0}, v_{0}\right)}(\zeta) d \eta
\end{aligned}
$$

We put

$$
T\left(w^{(\alpha)}(x, y)\right)=\frac{1}{2 \sqrt{2 \pi}} \int_{y}^{1} \int_{-\alpha}^{\alpha} \frac{1}{|\zeta|}\left[e^{(\eta-y)|\zeta|}-e^{(y-\eta)|\zeta|}\right] \widehat{f}_{\left(\eta, w^{(\alpha)}, v_{\varepsilon}\right)}(\zeta) e^{i \zeta x} d \zeta d \eta
$$

We shall prove

$$
\left\|T^{m}\left(w^{(\alpha)}(., y)\right)-T^{m}\left(w^{1(\alpha)}(., y)\right)\right\|_{L^{2}(\mathbb{R})}^{2} \leq\left(k^{2} e^{2 \alpha}\right)^{m} \frac{(1-y)^{m}}{m !}\left\|w^{(\alpha)}-w^{1(\alpha)}\right\| \|^{2}
$$

for every $\alpha>0, y \in(0,1), m \geq 1$ and $w^{(\alpha)}, w^{1(\alpha)} \in C\left([0,1] ; L^{2}(\mathbb{R})\right)$, where \|\|$\cdot \| \mid$ denotes sup-norm in $C\left([0,1] ; L^{2}(\mathbb{R})\right)$. 
We shall prove (51) by induction.

We define

$$
\chi_{[-\alpha, \alpha]}(\zeta)= \begin{cases}1 & |\zeta| \leq \alpha \\ 0 & |\zeta|>\alpha\end{cases}
$$

For $m=1$, noting that

$$
\widehat{T w^{(\alpha)}}(\zeta, y)=\frac{1}{2} \chi_{[-\alpha, \alpha]}(\zeta) \int_{y}^{1} \frac{1}{|\zeta|}\left[e^{(\eta-y)|\zeta|}-e^{(y-\eta)|\zeta|}\right] \widehat{f}_{\left(\eta, w^{(\alpha)}, v_{\varepsilon}\right)}(\zeta) d \eta
$$

we have

$$
\begin{aligned}
& \left\|T w^{(\alpha)}(., y)-T w^{1(\alpha)}(., y)\right\|_{L^{2}(\mathbb{R})}^{2}= \\
& =\left\|\widehat{T w^{(\alpha)}}(., y)-\widehat{T w^{1(\alpha)}}(., y)\right\|_{L^{2}(\mathbb{R})}^{2} \\
& =\frac{1}{4}\left\|\int_{y}^{1} \chi_{[-\alpha, \alpha]}(\zeta) \frac{e^{(\eta-y)|\zeta|}-e^{(y-\eta)|\zeta|}}{|\zeta|}\left(\widehat{f}_{\left(\eta, w^{(\alpha)}, v_{\varepsilon}\right)}(\zeta)-\widehat{f}_{\left(\eta, w^{1(\alpha)}, v_{\varepsilon}\right)}(\zeta)\right) d \eta\right\|_{L^{2}(\mathbb{R})}^{2} \\
& \leq \int_{-\infty}^{+\infty}\left|\int_{y}^{1} \chi_{[-\alpha, \alpha]}(\zeta) e^{(\eta-y)|\zeta|}\right| \widehat{f}_{\left(\eta, w^{(\alpha)}, v_{\varepsilon}\right)}(\zeta)-\widehat{f}_{\left(\eta, w^{1(\alpha)}, v_{\varepsilon}\right)}(\zeta)|d \eta|^{2} d \zeta \\
& \leq \int_{-\infty}^{+\infty}\left|\int_{y}^{1} e^{(\eta-y) \alpha}\right| \widehat{f}_{\left(\eta, w^{(\alpha)}, v_{\varepsilon}\right)}(\zeta)-\widehat{f}_{\left(\eta, w^{1(\alpha)}, v_{\varepsilon}\right)}(\zeta)|d \eta|^{2} d \zeta \\
& \leq e^{2 \alpha}(1-y) \int_{-\infty}^{+\infty} \int_{y}^{1}\left|\widehat{f}_{\left(\eta, w^{(\alpha)}, v_{\varepsilon}\right)}(\zeta)-\widehat{f}_{\left(\eta, w^{1(\alpha)}, v_{\varepsilon}\right)}(\zeta)\right|^{2} d \eta d \zeta \\
& \leq k^{2} e^{2 \alpha}(1-y) \int_{y}^{1} \int_{-\infty}^{+\infty}\left|w^{(\alpha)}(x, \eta)-w^{1(\alpha)}(x, \eta)\right|^{2} d x d \eta \\
& \leq e^{2 \alpha}(1-y) k^{2} \int_{y}^{1}\left\|w^{(\alpha)}(., \eta)-w^{1(\alpha)}(., \eta)\right\|_{L^{2}(\mathbb{R})}^{2} d \eta \\
& \leq e^{2 \alpha}(1-y) k^{2}\|\| w^{(\alpha)}-w^{1(\alpha)} \|\left.\right|^{2} .
\end{aligned}
$$

Therefore (53) holds. 
Suppose that (53) holds for $m=j$. We shall prove that (51) holds for $m=j+1$. We have

$$
\begin{aligned}
& \left\|T^{j+1}\left(w^{(\alpha)}(., y)\right)-T^{j+1}\left(w^{1(\alpha)}(., y)\right)\right\|_{L^{2}(\mathbb{R})}^{2}= \\
& =\left\|\widehat{T}\left(T^{j}\left(w^{(\alpha)}(., y)\right)\right)-\widehat{T}\left(T^{j}\left(w^{1(\alpha)}(., y)\right)\right)\right\|_{L^{2}(\mathbb{R})}^{2} \\
& \leq k^{2} e^{2 \alpha}(1-y) \int_{y}^{1}\left\|T^{j}\left(w^{(\alpha)}(., \eta)\right)-T^{j}\left(w^{1(\alpha)}(., \eta)\right)\right\|_{L^{2}(\mathbb{R})}^{2} d \eta \\
& \leq\left. k^{2} e^{2 \alpha}(1-y) \int_{y}^{1}\left(k^{2} e^{2 \alpha}\right)^{j} \frac{(1-\eta)^{j}}{j !}\left\|w^{(\alpha)}-w^{1(\alpha)}\right\|\right|^{2} d \eta \\
& \leq\left.\left(k^{2} e^{2 \alpha}\right)^{j+1} \frac{(1-y)^{j+1}}{(j+1) !}\left\|w^{(\alpha)}-w^{1(\alpha)}\right\|\right|^{2} .
\end{aligned}
$$

Therefore, by the induction principle, (53) holds for every $m$. From (53), we get

$$
\left.\left\|T^{m}\left(w^{(\alpha)}\right)-T^{m}\left(w^{1(\alpha)}\right)\right\|\right|^{2} \leq\left.\left(k^{2} e^{2 \alpha}\right)^{m} \frac{1}{m !}\left\|w^{(\alpha)}-w^{1(\alpha)}\right\|\right|^{2} .
$$

Since $\lim _{m \rightarrow \infty}\left(k^{2} e^{2 \alpha}\right)^{m} \frac{1}{m !}=0$, there exists a positive integer number $m_{0}$ such that

$$
\left(k^{2} e^{2 \alpha}\right)^{m_{0}} \frac{1}{m_{0} !}<1 .
$$

Hence $T^{m_{0}}$ is a contraction in $C\left([0,1] ; L^{2}(\mathbb{R})\right)$. It follows that the equation $T^{m_{0}}\left(w^{(\alpha)}\right)=$ $w^{(\alpha)}$ has a unique solution $w^{(\alpha)} \in C\left([0,1] ; L^{2}(\mathbb{R})\right)$.

We claim that $T\left(w^{(\alpha)}\right)=w^{(\alpha)}$. In fact, one has $T\left(T^{m_{0}}\left(w^{(\alpha)}\right)\right)=T\left(w^{(\alpha)}\right)$. Hence $T^{m_{0}}\left(T\left(w^{(\alpha)}\right)\right)=T\left(w^{(\alpha)}\right)$. By the uniqueness of the fixed point of $T^{m_{0}}$, one has $T\left(w^{(\alpha)}\right)=$ $w^{(\alpha)}$, i.e., the equation $T\left(w^{(\alpha)}\right)=w^{(\alpha)}$ has a unique solution $w^{(\alpha)} \in C\left([0,1] ; L^{2}(\mathbb{R})\right)$.

We have

$$
\begin{aligned}
4 & \left\|w_{0(y)}-w^{(\alpha)}(., y)\right\|_{L^{2}(\mathbb{R})}^{2} \\
= & \int_{-\alpha}^{\alpha}\left|\int_{y}^{1} \frac{\left[e^{(\eta-y)|\zeta|}-e^{(y-\eta)|\zeta|}\right]}{|\zeta|}\left[\widehat{f}_{\left(\eta, w_{0}, v_{0}\right)}(\zeta)-\widehat{f}_{(\eta, w}^{\left.(\alpha), v_{\varepsilon}\right)}(\zeta)\right] d \eta\right|^{2} d \zeta \\
& +\int_{|\zeta|>\alpha}\left|\int_{y}^{1} \frac{\left[e^{(\eta-y)|\zeta|}-e^{(y-\eta)|\zeta|}\right]}{|\zeta|} \widehat{f}_{\left(\eta, w_{0}, v_{0}\right)}(\zeta) d \eta\right|^{2} d \zeta
\end{aligned}
$$




$$
\begin{aligned}
& \leq 4 \int_{-\alpha}^{\alpha}\left|\int_{y}^{1} e^{(\eta-y)|\zeta|}\right| \widehat{f}_{\left(\eta, w_{0}, v_{0}\right)}(\zeta)-\widehat{f}_{\left(\eta, w^{(\alpha)}, v_{\varepsilon}\right)}(\zeta)|d \eta|^{2} d \zeta \\
& +4 \int_{|\zeta|>\alpha}\left|\int_{y}^{1} e^{(\eta-y)|\zeta|} \widehat{f}_{\left(\eta, w_{0}, v_{0}\right)}(\zeta) d \eta\right|^{2} d \zeta \\
& \leq 4 \int_{-\alpha}^{\alpha}\left|\int_{y}^{1} e^{(\eta-y) \alpha}\right| \widehat{f}_{\left(\eta, w_{0}, v_{0}\right)}(\zeta)-\widehat{f}_{\left(\eta, w^{(\alpha)}, v_{\varepsilon}\right)}(\zeta)|d \eta|^{2} d \zeta \\
& +4 e^{-2 y \alpha} \int_{|\zeta|>\alpha}\left|\int_{y}^{1} e^{(\eta-y)|\zeta|+y \alpha} \widehat{f}_{\left(\eta, w_{0}, v_{0}\right)}(\zeta) d \eta\right|^{2} d \zeta \\
& \leq 4 e^{-2 y \alpha}\left\{\int_{-\alpha}^{\alpha} \int_{y}^{1} e^{2 \eta \alpha}\left|\widehat{f}_{\left(\eta, w_{0}, v_{0}\right)}(\zeta)-\widehat{f}_{\left(\eta, w^{(\alpha)}, v_{\varepsilon}\right)}(\zeta)\right|^{2} d \eta d \zeta\right. \\
& \left.+\int_{|\zeta|>\alpha}\left|\int_{y}^{1} e^{(\eta-y)|\zeta|+y \alpha} \widehat{f}_{\left(\eta, w_{0}, v_{0}\right)}(\zeta) d \eta\right|^{2} d \zeta\right\} \\
& \leq 4 e^{-2 y \alpha}\left\{\int_{y}^{1} e^{2 \eta \alpha}\left(\int_{-\infty}^{+\infty}\left|f_{\left(\eta, w_{0}, v_{0}\right)}(\xi)-f_{\left(\eta, w^{(\alpha)}, v_{\varepsilon}\right)}(\xi)\right|^{2} d \xi\right) d \eta\right. \\
& \left.+\int_{|\zeta|>\alpha}\left|\int_{y}^{1} e^{|\zeta|+\alpha}\right| \widehat{f}_{\left(\eta, w_{0}, v_{0}\right)}(\zeta)|d \eta|^{2} d \zeta\right\} \\
& \leq 4 e^{-2 y \alpha}\left\{\int_{y}^{1} e^{2 \eta \alpha} \int_{-\infty}^{+\infty} k^{2}\left|v_{0}(\xi, \eta)-v_{\varepsilon}(\xi, \eta)+w_{0}(\xi, \eta)-w^{(\alpha)}(\xi, \eta)\right|^{2} d \xi d \eta\right. \\
& \left.+\int_{|\zeta|>\alpha} e^{2(|\zeta|+\alpha)} \int_{y}^{1}\left|\widehat{f}_{\left(\eta, w_{0}, v_{0}\right)}(\zeta)\right|^{2} d \eta d \zeta\right\} \\
& \leq 4 e^{-2 y \alpha}\left\{2 k^{2} \int_{y}^{1} e^{2 \eta \alpha}\left\|w_{0}(., \eta)-w^{(\alpha)}(., \eta)\right\|_{L^{2}(\mathbb{R})}^{2} d \eta\right. \\
& +2 \int_{0}^{1} \int_{-\infty}^{+\infty} e^{2 \alpha} k^{2}\left|v_{0}(\xi, \eta)-v_{\varepsilon}(\xi, \eta)\right|^{2} d \xi d \eta
\end{aligned}
$$




$$
\begin{aligned}
& \left.+\int_{|\zeta|>\alpha} e^{2(|\zeta|+\alpha)} \int_{y}^{1}\left|\widehat{f}_{\left(\eta, w_{0}, v_{0}\right)}(\zeta)\right|^{2} d \eta d \zeta\right\} \\
& \leq 4 e^{-2 y \alpha}\left\{2 k^{2} \int_{y}^{1} e^{2 \eta \alpha}\left\|w_{0}(., \eta)-w^{(\alpha)}(., \eta)\right\|_{L^{2}(\mathbb{R})}^{2} d \eta\right. \\
& +2 k^{2} e^{2 \alpha}\left\|v_{0}-v_{\varepsilon}\right\|_{2}^{2} \\
& \left.+\int_{|\zeta|>\alpha} e^{2(|\zeta|+\alpha)} \int_{y}^{1}\left|\widehat{f}_{\left(\eta, w_{0}, v_{0}\right)}(\zeta)\right|^{2} d \eta d \zeta\right\} .
\end{aligned}
$$

We put

$$
M_{1}=2 k^{2} e^{2 \alpha}\left\|v_{0}-v_{\varepsilon}\right\|_{2}^{2}
$$

and

$$
M_{2}=\int_{|\zeta|>\alpha} e^{2(|\zeta|+\alpha)} \int_{y}^{1}\left|\widehat{f}_{\left(\eta, w_{0}, v_{0}\right)}(\zeta)\right|^{2} d \eta d \zeta
$$

Therefore

$$
e^{2 y \alpha}\left\|w_{o(y)}-w^{(\alpha)}(., y)\right\|_{L^{2}(\mathbb{R})}^{2} \leq 2 k^{2} \int_{y}^{1} e^{2 \eta \alpha}\left\|w_{o}(., \eta)-w^{(\alpha)}(., \eta)\right\|_{L^{2}(\mathbb{R})}^{2} d \eta+M_{1}+M_{2} .
$$

Using Gronwall's inequality, we have

$$
e^{2 y \alpha}\left\|w_{o}(., y)-w^{(\alpha)}(., y)\right\|_{L^{2}(\mathbb{R})}^{2} \leq\left(M_{1}+M_{2}\right) e^{2 k^{2}(1-y)},
$$

hence

$$
\left\|w_{o}(., y)-w^{(\alpha)}(., y)\right\|_{L^{2}(\mathbb{R})}^{2} \leq e^{-2 \alpha y}\left(M_{1}+M_{2}\right) e^{2 k^{2}(1-y)} .
$$

From Theorem 2, we get

$$
\begin{aligned}
e^{-2 \alpha y} M_{1} & =2 e^{2(1-y) \alpha} k^{2}\left\|v_{0}-v_{\varepsilon}\right\|_{2}^{2} \\
& <2 D^{2} e^{2(1-y) \alpha} k^{2}\left(\ln \frac{1}{\varepsilon}\right)^{-2} .
\end{aligned}
$$

We have

$$
e^{-2 \alpha y} M_{2}=e^{-2 \alpha y} \int_{|\zeta|>\alpha} e^{2(|\zeta|+\alpha)} \int_{y}^{1}\left|\widehat{f}_{\left(\eta, v_{0}, w_{0}\right)}(\zeta)\right|^{2} d \eta d \zeta
$$




$$
\begin{aligned}
& \leq e^{-2 \alpha y} \int_{|\zeta|>\alpha} \int_{0}^{1} \frac{e^{6|\zeta|}}{e^{2 \alpha}}\left|\widehat{f}_{\left(\eta, v_{0}, w_{0}\right)}(\zeta)\right|^{2} d \eta d \zeta \\
& \leq e^{-2 \alpha(y+1)}\left\|e^{3|\zeta|}\left|\widehat{f}_{\left(\eta, v_{0}, w_{0}\right)}(\zeta)\right|\right\|_{2}^{2} .
\end{aligned}
$$

From (54)-(56) and choosing $\alpha=\frac{1}{2(1-y)} \ln \left(\ln \frac{1}{\varepsilon}\right)$, we get

$$
\begin{aligned}
\left\|w_{o}(., y)-w^{(\alpha)}(., y)\right\|_{L^{2}(\mathbb{R})}^{2} & <e^{2 k^{2}}\left[2 k^{2} D^{2}\left(\ln \frac{1}{\varepsilon}\right)^{-1}+\left\|e^{3|\zeta|}\left|\widehat{f}_{\left(\eta, v_{0}, w_{0}\right)}(\zeta)\right|\right\|_{2}^{2}\left(\ln \frac{1}{\varepsilon}\right)^{-1}\right] \\
& <C^{2}\left(\ln \frac{1}{\varepsilon}\right)^{-1} .
\end{aligned}
$$

Therefore

$$
\left\|w_{o}-w^{(\alpha)}\right\|_{2}<C\left(\ln \frac{1}{\varepsilon}\right)^{-1 / 2} .
$$

Denoting $w_{\varepsilon}=w^{(\alpha)}$ and $u_{\varepsilon}=v_{\varepsilon}+w_{\varepsilon}$, we get

$$
\begin{aligned}
\left\|u_{0}-u_{\varepsilon}\right\|_{2} & =\left\|w_{0}+v_{0}-v_{\varepsilon}-w_{\varepsilon}\right\|_{2} \\
& \leq\left\|w_{0}-w^{(\alpha)}\right\|_{2}+\left\|v_{0}-v_{\varepsilon}\right\|_{2} \\
& \leq C\left(\ln \frac{1}{\varepsilon}\right)^{-1 / 2}+D\left(\ln \frac{1}{\varepsilon}\right)^{-1} \\
& \leq(C+D)\left(\ln \frac{1}{\varepsilon}\right)^{-1 / 2} .
\end{aligned}
$$

This completes the proof of Theorem 3 .

\section{NUMERICAL RESULTS}

Let the problem

$$
\begin{aligned}
& \Delta u=f(x, y, u), x \in \mathbb{R}, y>1 \\
& u(x, 1)=\varphi(x)
\end{aligned}
$$

with

$$
\begin{aligned}
& f(x, y, u)=\arctan \left(|u|+x^{2}+y^{2}\right)+g(x, y) \\
& g(x, y)=\frac{4 C x}{\left(x^{2}+4\right)^{3}}+\frac{-2 C}{\left(x^{2}+4\right)^{2}}-\arctan \left(\frac{C}{x^{2}+4}+x^{2}+y^{2}\right), C=\frac{4}{\sqrt{2 \pi}} \\
& \varphi(x)=\frac{C}{4+x^{2}}
\end{aligned}
$$


The exact solution of (57) is $u(x, y)=\frac{C}{x^{2}+4}, C=4 / \sqrt{2 \pi}$ and we solve numerically this problem for $x \in \mathbb{R}, y>1$ by using the iterative sequence defined by

$$
\begin{array}{r}
u^{(n+1)}(x, y)=-\int_{-\infty}^{\infty} \int_{1}^{\infty} N(x, y, \xi, \eta) f\left(\xi, \eta, u^{(n)}(\xi, \eta)\right) d \xi d \eta+ \\
\int_{-\infty}^{\infty} N_{y}(x, y, \xi, 1) \varphi(\xi) d \xi
\end{array}
$$

and whose graphic is displayed in Fig.1 on the interval $[-4,4] \times[1,4]$.

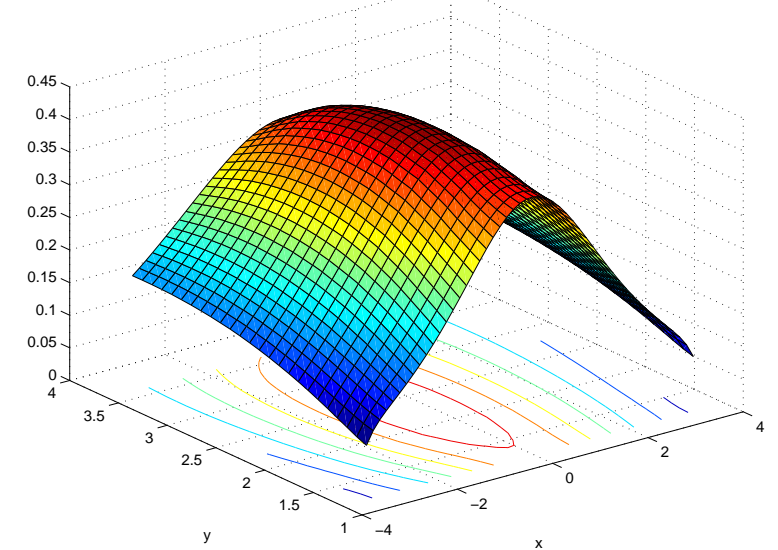

Fig.1: approximated solution

For the second problem

$$
\begin{aligned}
& \Delta v=0, x \in \mathbb{R}, 0<y<1 \\
& v(x, 1)=\varphi(x), v_{y}(x, 1)=0
\end{aligned}
$$

whose exact solution is $v(x, y)=\frac{1}{2 \sqrt{2 \pi}}\left[\frac{3-y}{x^{2}+(3-y)^{2}}+\frac{1+y}{x^{2}+(1+y)^{2}}\right]$, we calculate the regularized solution $v_{\varepsilon}(x, y)$ of (58) for $\varepsilon=10^{-2}$ by the formula (51).

Finally for the third problem

$$
\begin{aligned}
& \Delta w=f(x, y, v+w), x \in \mathbb{R}, 0<y<1 \\
& w(x, 1)=0, w_{y}(x, 1)=0
\end{aligned}
$$

the regularized solution $w_{\varepsilon}(x, y)$ of the problem (59) is calculated from its definition (52). So in Fig. 2 we have drawed the regularized solution of the problem 2 i.e. $u_{\varepsilon}(x, y)=v_{\varepsilon}(x, y)+$ $w_{\varepsilon}(x, y)$ on the interval $[-4,4] \times[0,1]$. 


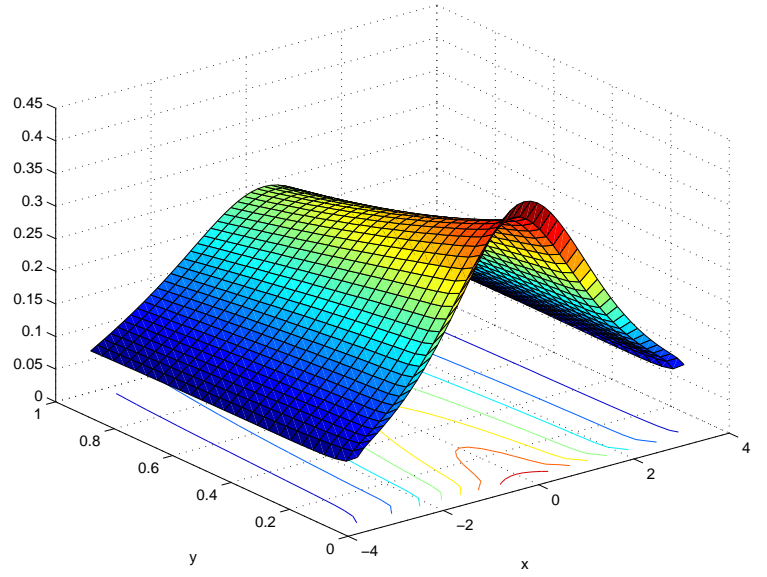

Fig.2: regularized solution

For comparison Fig.3 gives the exact solution on the interval $[-4,4] \times[0,4]$.

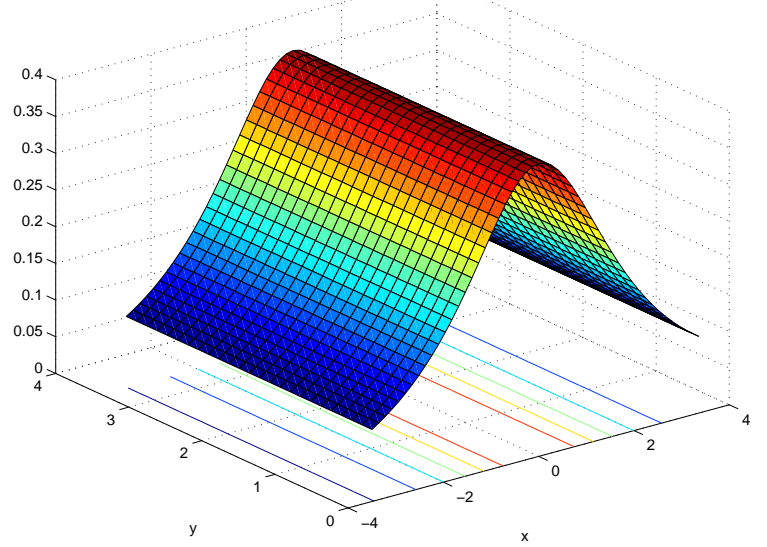

Fig.3: exact solution

\section{REFERENCES}

[AS] Andersen, R. S. and Saull, V. A., Surface temperature history determination from bore hole measurements, Journal of International Association Mathematical Geology, 5, pp. 269-283, 1973.

[B] Bourgeois, L., A mixed formulation of quasi-reversibility to solve the Cauchy problems for Laplace's equations, Inverse Problems 21, No. 3, pp. 1087-1104, 2005.

[BBC] Beck, J. V., Blackwell, B. and Clair, C. R. St., Inverse Heat Conduction, Ill-posed Problem, Wiley, New York, 1985.

[C] Colton, D., Partial differential equations, Random House, New York, 1988.

[C1] Cavazzoni, R., On the Cauchy problem for elliptic equation in a disk, Rend. Circ. Mat. Palermo (2) 52, No. 1, pp. 131-140, 2003.

[CHWY] Cheng, J., Hon, Y. C., Wei, T. and Yamamoto, M., Numerical computation of a Cauchy problem for Laplace's equations, ZAMM Z. Angew. Math. Mech. 81, No. 10, pp. 665-674, 2001. 
[HQ] Huang, Y. and Quan, Z., Regularization for a class of ill-posed Cauchy problems, Proc. Amer. Math. Soc., Vol. 133, No. 10,pp. 3005-3012, 2005.

[HR] Han, H. and Reinhardt, H. J., Some stability estimates for Cauchy problems for elliptic equations, J. Inverse Ill-Posed Probl. 5, No. 5, pp. 437-454, 1997.

[HR1] Hao, D. N. and Reinhardt, H. J., On a sideways parabolic equation, Inverse Problems, 13, pp. 297-309, 1997.

[HRS] Hao, D. N., Reinhardt, H. J. and Schneider, A., Numerical solution to a sideways parabolic equation, International Journal for Numerical methods in Engineering, 50, pp. 1253-1267, 2001.

[KS] Klibanov, M. V. and Santosa, F. V.,A computational quasi-reversibility method for Cauchy problems for Laplace's equation, SIAM J. Appl. Math. 51, No. 6, pp. 1653-1675, 1991.

[K] Krylov, A. L., The Cauchy problem for Laplace's equations in the complex domain, Dokl. Akad. Nauk SSSR 188, pp. 748-751, 1969.

[L] Levine, H. A., Continuous data dependence, regularization and a three lines theorem for the heat equation with data in a space like direction, Annali di Matematica Pura ed Applicata, CXXXIV, pp. 267-286, 1983.

[LV] Levine H. A. and Vessella S., Estimates and regularization for solutions of some illposed problems of elliptic and parabolic type, Rediconti del Circolo Matematica di Palermo, 34, pp. 141-160, 1985.

[M] Murio, D., The mollification method and the numerical solution of ill-posed problems, Wiley, New York, 1993.

[QD] P. H. Quan and N. Dung, A backward nonlinear heat equation: regularization with error estimates, accepted for publication in Applicable Analysis, 2005.

[QT] P. H. Quan and D. D. Trong, Temperature Determination from Interior Measurements: the Case of Temperature Nonlinearly Dependent Heat Source, Vietnam Journal of Math., 32, pp. 131-142, 2004.

[T] Tautenhahn, U., Optimal stable solution of Cauchy problems for elliptic equations, Z. Anal. Anwendungen 15, No. 4, pp. 961-984, 1996.

[TT] D. D. Trong and N. H. Tuan, Regularization and error estimates for nonhomogeneous backward heat problems, Electronic J. of Diff. Eq. Vol. 2006, No. 04, pp. 1-10, 2006. 